\title{
Vibronic Magnetoelectric Effects in the Bi-Based Multiferroics
}

\author{
P. KONSIN* AND B. SORKIN \\ Institute of Physics, University of Tartu, Riia 142, 51014 Tartu, Estonia
}

\begin{abstract}
The detailed magneto-electron-lattice (vibronic) theory of ferroelectricity in the Bi-based multiferroics $\left(\mathrm{BiFeO}_{3}\right.$ etc.) is developed further. The vibronic admixture of the empty oxygen $2 p$ states to the Bi $6 s$ lone electron pair state leads to ferroelectricity. These interactions are the driving and restoring forces of the structural phase transformations of the Bi-based multiferroics. The free energy of the $\mathrm{BiFeO}_{3}$-type multiferroics with the ferroelectric and the $G$-type antiferromagnetic phase transitions are derived. The Zeeman splitting and spintransversal optical phonon couplings are also important.
\end{abstract}

DOI: $10.12693 /$ APhysPolA.127.243

PACS: 63.20.kd, 77.84.-s, 77.80.Bh, 77.80.-e

\section{Introduction}

Multiferroics are multifunctional materials, in which two or more order parameters (ferroelectric, ferro(antiferro) magnetic and ferroelastic) coexist in a single compound [1-5]. The vibronic theory of ferroelectric displacive phase transitions with an order-disorder component in the $\mathrm{BaTiO}_{3}$-type perovskite oxides is developed, see [6-8]. In [9] a vibronic approach is proposed for explaining of ferroelectric properties of the Bi-based multiferroics.

In this contribution we take into account vibronic and magnetic couplings in the $\mathrm{BiFeO}_{3}$-type multiferroics. Magnetism and ferroelectricity are involved with local spins (Fe, Mn) and off-center structural distortions (Bi), respectively $[1-5,9]$. We obtained the free energy of the Bi-based multiferroics in external electric and magnetic fields. On the base of this free energy the magnetoelectric effects can be studied in this type material.

\section{Free energy}

Using the results of the papers $[9,10]$ we obtained the free energy of the $\mathrm{BiFeO}_{3}$-type multiferroics

$$
F=F_{\mathrm{e}}+F_{\mathrm{m}},
$$

where $F_{\mathrm{e}}$ is the density of the free energy of the ferroelectric subsystem and $F_{\mathrm{m}}$ is the free energy of the magnetic subsystem including direct magnetoelectric couplings [10].

In comparison with [9] in [11] and in this paper all actual vibronic couplings, including the electron-lattice vertex corrections and phonon-phonon couplings are taken into account. The influence of the magnetic field $\boldsymbol{H}$ on the electronic states is considered. We have

$$
\begin{aligned}
F_{\mathrm{e}} & =F_{0}+\left[\frac{1}{N} V^{2}\left(\alpha_{1}+\alpha_{2} \Delta\right)+\frac{M \bar{\omega}_{0}^{2}}{2}\right]\left(x^{2}+y^{2}\right) \\
& +\left[\left(\alpha_{1}+\alpha_{2} \Delta\right) \frac{\left(V^{2}+V_{\beta}^{2}\right)}{N}+\frac{M \bar{\omega}_{0}^{2}}{2}+\frac{1}{2} k_{0 \beta}\right] z^{2}
\end{aligned}
$$

*corresponding author; e-mail: konsin@fi.tartu.ee

$$
\begin{aligned}
& +\left[\left(\beta_{1}+\beta_{2} \Delta\right) \frac{\left(V^{2}+V_{\beta}^{2}\right)^{2}}{N^{2}}\right] z^{4}-\frac{\mathrm{e}_{\beta}^{\perp}}{\sqrt{N}} \eta_{\perp} E_{\perp} \\
& -\frac{Q^{*}}{\sqrt{N}} z E_{\|}-V^{2} \frac{\left(\mu_{\mathrm{B}} H\right)^{2} \kappa_{1,2}}{2 \Delta^{3}}\left(x^{2}+y^{2}\right) \\
& -\left(V^{2}+V_{\beta}^{2}\right) \frac{\left(\mu_{\mathrm{B}} H\right)^{2} \kappa_{1,2}}{2 \Delta^{3}} z^{2} .
\end{aligned}
$$

The vibronic coupling constants

$$
V=\left\langle\psi\left({ }^{1} A_{1}^{\prime}\left(\mathrm{Bi} 6 s^{2}\right)\right)\left|\frac{\delta H}{\delta x}\right| \psi\left(p_{x}(\mathrm{O})\right)\right\rangle
$$

and

$$
V_{\beta}=\left\langle\psi\left({ }^{1} A_{1}^{\prime}\left(\mathrm{Bi} 6 s^{2}\right)\right)\left|\frac{\delta H}{\delta \tau_{\beta}}\right| \psi\left(R_{15}(\mathrm{O})\right)\right\rangle .
$$

The quantity $\tau_{\beta} \approx z[9]$ and $x, y, z$ are the Cartesian coordinates, $N$ is the number of unit cells, $\Delta$ is the averaged gap between the bands. The coefficients: $\alpha_{1}=\alpha_{10} f_{s} \frac{N_{0}}{\Delta}$, $\alpha_{2}=\alpha_{20} f_{s} \frac{N_{0}}{\Delta^{2}}, \beta_{1}=\beta_{10} f_{s} \frac{N_{0}}{\Delta^{3}}$ and $\beta_{2}=\beta_{20} f_{s} \frac{N_{0}}{\Delta^{4}}\left(N_{0}\right.$ is the number of electrons in the bands). The calculated in [9] coefficients are the following: $\alpha_{10}=-0.4131$, $\alpha_{20}=-0.6498, \beta_{10}=17.6455$ and $\beta_{20}=-10.29$. The quantity $\bar{\omega}_{0}$ is the frequency of the potential optical vibrations with the wave vector $\boldsymbol{q}=0$ renormalized by the phonon-phonon couplings and the contribution from higher order electron-phonon interactions (i.e., vertex corrections)

$$
\begin{gathered}
M \bar{\omega}_{0}^{2}=M \omega_{0}^{2}+12 \sum_{\boldsymbol{q}} \sum_{i=1,2} \frac{B\left(\begin{array}{cccc}
0 & 0 & \boldsymbol{q} & -\boldsymbol{q} \\
1 & 1 & i & i
\end{array}\right)}{M_{i} \omega_{i \boldsymbol{q}}^{2}}\left(n_{i \boldsymbol{q}}+\frac{1}{2}\right) \\
+M^{-1} \frac{V^{4}}{\Delta^{3}} \sum_{\boldsymbol{q}} \frac{\hbar}{\omega_{\boldsymbol{q}}} \operatorname{coth} \frac{\hbar \omega_{\boldsymbol{q}}}{k_{\mathrm{B}} T}+\frac{V_{\beta}^{4}}{\kappa_{0 \beta} \Delta^{3}} k_{\mathrm{B}} T
\end{gathered}
$$

Here, $M$ is the mass factor, the index 1 belongs to the transversal optical (TO) and 2 to the transversal acoustic (TA) branches, $B$ corresponds to the 4 th order anharmonicity (renormalized in respect of the 3 rd order one), $\kappa_{0 \beta}$ is the corresponding force constant, $\omega_{0}$ is the bare phonon frequency of the active long wave transverse optical vibration. The $g$-factors corresponding to the Zee- 
man effect in Eq. (2) for the valence band are $g_{\mathrm{v}}$ and the conduction band $g_{\mathrm{c}}^{(1,2,3)}$.

Here [11]:

$$
\begin{gathered}
\kappa_{1}=\kappa_{2}=\frac{1}{2}\left(g_{\mathrm{c}}^{(1)}-g_{\mathrm{v}}\right)^{2}+\frac{1}{6}\left(g_{\mathrm{c}}^{(2)}-g_{\mathrm{v}}\right)^{2} \\
++2 t^{2}-\frac{4}{\sqrt{18}} t\left[g_{\mathrm{c}}^{(2)}+g_{\mathrm{c}}^{(3)}+2 g_{\mathrm{v}}\right] .
\end{gathered}
$$

The dynamic Born effective charge, $Q^{*}$ equals [9, 11]:

$$
Q^{*}=Q+\Delta Q \frac{a_{0} \sqrt{N}}{z_{0}} .
$$

Here, $a_{0}$ is the cell constant, $Q$ is the ionic charge and $\frac{z_{0}}{\sqrt{N}}$ is the ionic displacement $\left(B i^{3+}\right)$. We obtained in [11]:

$$
\Delta Q=\frac{2|e|}{N} \sum_{\boldsymbol{k}} \frac{V(\boldsymbol{k}) \frac{z_{0}}{\sqrt{N}}}{E_{p}(\boldsymbol{k}+\boldsymbol{q})-E_{s}(\boldsymbol{k})}\left(f_{s}-f_{p}\right),
$$

where $E_{p, s}$ are the renormalized by the vibronic coupling [9] electronic spectra of the $\mathrm{Bi} 6 \mathrm{~s}^{2}$ state and $2 p$ states of $\mathrm{O}$, correspondingly, $f_{s}$ and $f_{p}$ are the band filling numbers. The quantity in Eq. (2) $\eta_{\perp}=\sqrt{x^{2}+y^{2}}$ and the electric fields $E_{\perp, \|}\left(E_{\perp}: \boldsymbol{E}\right.$ perpendicular to the caxis, $\left.E_{\|}: \boldsymbol{E} \| c\right)$. A certain part of the oxygen $p$ orbital is admixtured to $\mathrm{Bi} 6 \mathrm{~s}^{2}$ states. We have calculated for the spontaneous polarization of $\mathrm{BiFeO}_{3} P_{s}=6.02 \mu \mathrm{C} / \mathrm{cm}^{2}$, close to the measured in [12]. For the $\mathrm{BiFeO}_{3}$ films higher values of $P_{s}$ are obtained. In $\mathrm{BiFeO}_{3}-\mathrm{PbTiO}_{3}-$ based multiferroics-ceramics [10] significantly enhanced multiferroic properties are established. Favouring of the ferroelectric order takes place. It is supported by the vibronic coupling covalent hybridization of the $\mathrm{Pb} 6 \mathrm{~s}^{2}$ electronic states with the oxygen $2 p$ states which leads to the displacement of the equilibrium positions of the $\mathrm{Pb}$ ions. At this, the free energy of the ferroelectric subsystem analogous to Eq. (2) can be obtained. For attempts to understand magnetoelectric effects the free energy $F_{\mathrm{m}}$ can be used (see also [10]):

$$
F_{\mathrm{m}}=F_{\text {exch }}+F_{\mathrm{L}}+F_{\text {an }}+F_{\mathrm{M}} \text {, }
$$

where $F_{\text {exch }}$ is the inhomogeneous exchange energy, $F_{\mathrm{L}}$ is the magnetoelectric coupling that is linear in gradient, $F_{\text {an }}$ is the magnetic anisotropy energy, and $F_{\mathrm{M}}$ is the magnetic energy [10]. The influence of the spin-TO phonon coupling on the magnetic properties of the $\mathrm{Bi}$ based multiferroics using the Heisenberg model for antiferromagnetic properties can be calculated.

\section{Acknowledgments}

The research was supported by the Estonian Research Council (project IUT2-27) and by the European Union through the European Regional Development Fund (projects 3.2.0101.11-0029 and SF0180013s07AP).

\section{References}

[1] G.A. Smolenskii, I.E. Chupis, Usp. Fiz. Nauk 137, 415 (1982).

[2] W. Eerenstein, N.D. Mathur, J.F. Scott, Nature 4472, 759 (2006).

[3] S.-W. Cheong, M. Mostovoy, Nature Mater. 6, 13 (2007).

[4] M.J. Fiebig, Phys. D Appl. Phys. 38, R123 (2005).

[5] R. Ramesh, N.A. Spaldin, Nature Mater. 6, 21 (2007).

[6] P. Konsin, N. Kristoffel, in: Interband Model of Ferroelectrics, Eds. E. Bursian, Y. Girshberg, Hertzen Pedagogical Institute, Leningrad 1987, p. 32.

[7] N. Kristoffel, P. Konsin, Phys. Status Solidi B 149, 11 (1988) and references therein.

[8] I.B. Bersuker, The Jahn-Teller Effect, Cambridge University Press, Cambridge 2006.

[9] P. Konsin, B. Sorkin, Integrat. Ferroelectr. 109, 81 (2009).

[10] N. Wang, J. Chang, A. Pyatakov, A.K. Zvezdin, J.F. Li, L.E. Cross, D. Viehland, Phys. Rev. B $\mathbf{7 2}$ 104434 (2005).

[11] P. Konsin, B. Sorkin, Ferroelectrics 418, 100 (2011).

[12] J.R. Teague, R. Gerson, W.J. James, Solid State Commun. 8, 1073 (1970). 